\title{
The spatial frame of reference in object naming and discrimination of left-right reflections
}

\author{
PATRICIA A. MCMULLEN and PIERRE JOLICOEUR \\ University of Waterloo, Waterloo, Ontario, Canada
}

\begin{abstract}
The effects of stimulus rotation and observer's head-tilt position on various pattern-recognition tasks were investigated to compare the external directions most closely aligned with the spatial frame of reference. Specifically, the effects of these factors on the time to name objects were compared with their effects on the time to discriminate left-facing from right-facing lateral views of these objects, as well as with their effects on the time to discriminate normal from mirrorimaged alphanumeric characters. The naming task relied upon a reference frame more closely aligned with retinal directions than with environmental directions. In contrast, both discrimination tasks relied upon a frame aligned more closely with environmental directions. Overall, the nature of the task exerts a greater influence on the directions with which the frame of reference is aligned than do the stimulus attributes.
\end{abstract}

Rotating an object in the frontal plane can have minimal effects on the time to identify or name that object (Cooper \& Shepard, 1973; Corballis \& Nagourney, 1978; Corballis, Zbrodoff, Shetzer, \& Butler, 1978; Eley, 1982; White, 1980). This pattern of results tends to be found when familiar, relatively simple stimuli, such as letters and digits, are shown repeatedly throughout the experiment. However, three independent investigators recently have demonstrated that the time to name line drawings of objects increases linearly when the objects are rotated up to $120^{\circ}$ from the environmental upright, provided that the same objects are not repeated (Jolicoeur, 1985, 1988; Maki, 1986; Shwartz, 1981). These results demonstrate that the representations of objects, or the processes that map visual stimuli onto these representations, or both, can be sensitive to the orientation of stimulus patterns.

The concept of a spatial reference frame has been invoked to account for the effects of stimulus orientation in a variety of spatial tasks (e.g., Attneave \& Olson, 1967; Attneave \& Reid, 1968; Corballis, Nagourney, Shetzer, \& Stefanatos, 1978; Corballis \& Roldan, 1975; Corballis, Zbrodoff, \& Roldan, 1976; Palmer, 1985; Robertson, Palmer, \& Gomez, 1987; Rock, 1973). A reference frame is a process or structure that often is conceptualized as an orthogonal grid used to assign directions (top, bottom, and sides) in space or to a mental representation (Palmer, 1985).

This research was supported by Grant A2579 from the Natural Sciences and Engineering Research Council of Canada awarded to Pierre Jolicoeur. This research constituted partial fulfillment of the requirements for the PhD degree granted by the University of Waterloo to the first author. We gratefully acknowledge the assistance of Margaret Ingleton and the comments provided by $M$. C. Corballis and two anonymous reviewers. Correspondence may be addressed to Patricia A McMullen, who is now at the Department of Psychology, CarnegieMellon University, Pittsburgh, PA 15213.

-Accepted by previous editor, Alice F. Healy
The now classic spatial task to demonstrate an effect of stimulus orientation is the discrimination of mirrorimaged and normal versions of a form (Cooper \& Shepard, 1973; Corballis, Nagourney, et al., 1978). In this task, the effects of stimulus orientation on response time have been assumed to reflect the time to align the top of an image of the visual stimulus with the top of a spatial reference frame. ${ }^{1}$ The top of the frame of reference is assumed to correspond to the top of the object representation. Some investigators have sought to determine the upright orientation of the reference frame used during these discriminations (Corballis et al., 1976; Corballis, Nagourney, et al., 1978). Two different upright orientations have been compared: one in which the upright of the frame is most closely aligned with the environmental upright ${ }^{2}$ and another in which it is most closely aligned with the retinal upright. Normally, these two upright orientations are confounded. However, tilting a subject's head during task performance dissociates the environmental from the retinal upright (e.g., see Corballis, Nagourney, et al., 1978; see also Rock, 1973).

Suppose that response times are fastest when stimuli are perfectly aligned with the perceptual frame of reference. In addition, suppose, to a first approximation, that the degree of misalignment between the orientation of the stimulus and the orientation of the frame produces a linear increase in response time. On the basis of these assumptions, it is possible to derive a pattern of response times across stimulus orientations that depends on a single parameter: the upright orientation of the subjective reference frame (Corballis, Nagourney, et al., 1978; Corballis \& Roldan, 1975; Corballis et al., 1976). For example, when the head is tilted, a response pattern in which upright stimuli relative to the environment are responded to most quickly, with increasing response times as the stimuli are rotated away from this position, is consistent with the use of an environmentally aligned frame. On the 
other hand, a pattern in which stimuli aligned with the head are responded to most quickly, with increasing response time as stimulus and head are misaligned, suggests a retinally aligned reference frame.

Rock and his colleagues (Rock, 1973, 1974; Rock \& DiVita, 1987; Rock \& Heimer, 1957) have proposed that rotated forms are perceived within a reference frame that is aligned predominantly with the environmental upright. In this view, generally, a form is corrected from initial retinal directions to environmental directions. However, under conditions of high stimulus complexity or low stimulus familiarity, this general correction does not occur because some visual capacity (Rock, 1986) or processingspeed deadline is exceeded due to "too many subparts and relationships among such parts" (Rock, 1973, p. 128) in the form. Therefore, Rock would predict that complex and unfamiliar forms are perceived within retinally aligned frames of reference. All other forms would be perceived within environmentally aligned frames.

In support of this view, mirror-normal discriminations of familiar, relatively simple letters and digits with the head tilted have most strongly indicated the use of an environmentally aligned reference frame (Corballis, Nagourney, et al., 1978; Corballis et al., 1976). As well, judgments about the symmetry or repetition of unfamiliar, relatively complex random-dot patterns located on both sides of a vertical, horizontal, or $45^{\circ}$ oblique line produce a performance pattern that is clearly consistent with the use of a retinally aligned reference frame (Corballis \& Roldan, 1975; Corballis et al., 1976). These results are consistent with the view that stimulus attributes such as complexity and familiarity influence the spatial reference frame (Corballis et al., 1976; Rock, 1973, 1974). However, task and stimulus set are confounded in these studies. Therefore, it is possible that different frames of reference are used, depending on the task.

In addition to the possible influence of stimulus and task attributes on the frame is evidence that subjects have some ability to control which reference frame is used in recognition. When subjects were informed of the orientation of tilted fragmented objects prior to each trial block, recognition was better for retinally aligned than for environmentally aligned stimuli (Rock \& Heimer, 1975, Experiment 6). In contrast, without these instructions, environmentally aligned stimuli were recognized better than retinally aligned stimuli. Also, mirror-normal discriminations of letters and digits were performed within a reference frame more closely aligned with retinal directions when subiects were instructed to consider the upright, as defined by a border around the display that was tilted with the retina (Corballis, Nagourney, et al., 1978). Again, in the absence of these instructions, the frame of reference was more closely aligned with environmental than retinal directions.

In summary, previous research suggests that a number of factors can influence the perceptual frame of reference within which effects of stimulus orientation take place.
These factors include the nature of the task (Corballis et al., 1976; Hock \& Sullivan, 1981), stimulus attributes and presentation conditions (Corballis, Nagourney, et al., 1978; Corballis et al., 1976; Rock, 1973), and instructions (Attneave \& Olson, 1967; Attneave \& Reid, 1968; Corballis, Nagourney, et al., 1978; Rock, 1973; Rock \& Heimer, 1957).

In the present experiments, we investigated the frame of reference in a task that is now known to show effects of stimulus orientation: object naming. In the absence of any instructional bias, performance of this task was compared with other tasks to determine whether properties of the stimuli or the task per se most influence the direction with which the reference frame is aligned.

Much of the previous work on the perception of rotated objects has employed recognition-memory tasks. Although recognition memory and naming performance may reflect the same orientation-sensitive mechanisms, the objectnaming task may be a more appropriate tool to investigate these mechanisms for the following reasons. The processes involved in object naming may be more closely related to the processes involved in object recognition than are those involved in recognition-memory tasks. Naming is more likely to involve mapping a broad class of visual stimuli onto long-term representations, whereas recognition memory may reflect matching to memory for a single "episodic" trace. Naming real objects also exclusively involves decision matches between visual input and stored representations, unlike recognition memory, which involves both matches and mismatches. Since the mechanisms involved in matches are of primary concern, naming is a more efficient task and yields a useful measure on every trial.

In addition, reaction time was used as the primary measure of naming performance in the present study. This continuous measure reflects online efficiency of all processing stages for each trial in which a correct match is made. In contrast, accuracy, which has been the measure used in recognition memory, is a discrete measure that reflects only the final success or failure of the matching process. Also, naming times can be compared with reaction times in other tasks, such as mirror-normal discrimination, which show effects of stimulus orientation with this measure.

\section{EXPERIMENT 1}

The purpose of this experiment was to discover the directions (retinal or environmental) with which the reference frame is most closely aligned when rotated objects are named. Each subject named line drawings of rotated objects with his/her head in an upright and a tilted position. Similarly, the subjects made mirror-normal discriminations of rotated letters and digits, as a control condition known to involve a frame predominantly aligned with environmental directions (see Corballis, Nagourney, et al., 1978; Corballis et al., 1976). 


\section{Method}

\section{Subjects}

Fifty-two paid participants were recruited from the undergraduate population at the University of Waterloo. The data from 48 of these subjects ( 24 females) were included in the final analysis, with the remaining 4 subjects failing to meet an accuracy criterion (described later). All subjects had normal or corrected-to-normal vision and spoke English as their first language. No subject participated in any of the other experiments reported in this paper.

\section{Apparatus and Stimuli}

The experiment was programmed and conducted with a microcomputer that controlled two slide projectors fitted with electronic shutters. Thirty-five-millimeter slides of objects or letters and digits were rear-projected onto a mylar screen that was framed by a matt-black rectangular border. The screen, object, and letter/digit stimuli subtended approximate visual angles of $6.2^{\circ}, 3.6^{\circ}$, and $2.6^{\circ}$, respectively. The original stimuli were photoreduced and photocopied onto translucent mylar film that was then cut and fit into 35-mm slide mounts.

One hundred twenty two-dimensional line drawings of objects with a distinct top and bottom (horizontally asymmetric) were used as stimuli (Snodgrass \& Vanderwart, 1980; see Appendix A). All stimuli were rotated from the environmental vertical, in the frontal plane, in $60^{\circ}$ clockwise steps, ranging from $0^{\circ}$ to $300^{\circ}$. In addition, 96 items for mirror-normal discriminations were produced from eight asymmetric, alphanumeric characters in Helvetica font (F, G, L, P, R, J, 5, and 7). These items were rotated in six orientations and were either mirror-reflected or normal in format.

The subjects responded to the stimuli either by pressing one of two telegraph keys (in the mirror-normal discrimination task) or by speaking into a microphone connected to a voice-activated relay (in the naming task). Reaction time was measured from the opening of the shutter to the subject's response and was recorded by the computer to the nearest millisecond.

In addition, a custom-designed head-tilt apparatus maintained each subject's head in either an upright position or tilted to the left or the right. This apparatus was a metal frame with two parts. One part included a chin rest and padded reinforcers that could be adjusted to rest against the subject's temples. This part of the frame pivoted just below the chin and could be rotated to any position within $90^{\circ}$ from upright. The other part of the apparatus was the base and was bolted to the table in front of the subject. Whenever a new head-tilt condition was introduced, the tilting apparatus was repositioned laterally to maintain alignment of the center of the subject's head with the center of the screen.

In the head-tilt conditions, the head was tilted $66^{\circ}$ to either side to achieve retinal meridian tilts of approximately $60^{\circ}$. An additional head tilt of about $10 \%\left(6^{\circ}\right)$ of the desired retinal tilt was included to compensate for the counterrolling of the eyeballs due to the vestibular-ocular reflex (Corballis et al., 1976; Miller, 1962).

\section{Procedure}

Each subject was tested individually, and half of the subjects performed the naming task before the mirror-normal discriminations. The entire session lasted approximately $1 \mathrm{~h}$. The subjects were given a set of written instructions appropriate to each upcoming task that emphasized both speed and accuracy. All trials began with a central fixation point displayed for $1 \mathrm{sec}$. The fixation point was immediately followed by a stimulus presentation that lasted until a response had been made or until $4 \mathrm{sec}$ had elapsed.

Naming. For the naming task, the subjects were instructed to enunciate clearly the most appropriate name for each object into a microphone mounted in front of them. A new trial was initiated by the experimenter with a button press. After each trial, the experimenter coded the subject's response on the computer as correct, incorrect, or spoiled. Hesitations, such as "ah," or any other pre- mature tripping of the voice key constituted a spoiled trial. Naming responses for a particular object that did not correspond to any of the acceptable names listed in Appendix A were coded as incorrect.

The subjects named 60 objects each, with their heads upright. All subjects also named a different set of 60 objects with their heads tilted. Half of the subjects had their heads tilted left and half had their heads tilted right. Half of the subjects performed with their heads upright before they performed with their heads tilted. The other subjects had the reverse tilt-condition order. Each subject saw 10 objects at one particular stimulus orientation under each headtilt condition.

The first 24 subjects had only one practice session of 16 trials at the beginning of the naming session with the head upright. After the first 24 subjects were run, the slides were left-right reversed in the slide trays to avoid object-specific effects on orientation. Therefore, objects that were shown at an orientation of $60^{\circ}$ for the first 24 subjects subsequently were shown at $300^{\circ}$, those at $120^{\circ}$ were flipped to $240^{\circ}$, and vice versa. Objects at $0^{\circ}$ and $180^{\circ}$ rotation were not changed. For the final 24 subjects, 16 practice trials on objects not shown in the experimental trials were presented before each new head-tilt condition, with the head in the position of the upcoming experimental trials

Six sets of 120 objects, with 20 different objects at each of six orientations, were created such that every object appeared once in each set in a different orientation. Equal numbers of objects known to produce slow and fast naming responses when averaged across all orientations (as determined by a pilot study) were included at each orientation in each set to control for the effect of item differences on the effects of orientation. No more than four consecutive trials were allowed to have the same orientation.

The order of presentation of sets across subjects was determined by a Latin square. Each subject named the first 60 objects in the assigned set in one head-tilt position and the final 60 objects in the other head-tilt position. Half of the subjects in each of the two headtilt groups (upright and left, upright and right) were males, and gender was also counterbalanced with object set. In addition, headtilt group and tilt-condition order were counterbalanced with set. In this way, every set was shown in every head-tilt-position combination (i.e., upright and left, upright and right, left and upright, and right and upright) twice.

Mirror-normal discriminations. Each subject also performed 96 mirtor-normal discriminations under each of the three head-tilt positions. Responses to normally formatted characters were made by pressing a key located under the index finger of the dominant hand. Similarly, responses to mirror-image characters were made by pressing a key located under the index finger of the nondominant hand. The reaction time and response were recorded by the computer for each trial. The intertrial interval was 1,200 msec. Practice trials at the onset of the entire discrimination session consisted of the last 16 experimental trials in the first head-tilt-position session and were performed with head upright.

Six sets of 16 trials were created by random assignment with the constraint that the same character, orientation, or format appeared in no more than four consecutive trials. For each subject, all six sets were presented in each head-tilt position, which meant that each character was presented once in each orientation and format. A Latin square determined the presentation order of these object sets and the head-tilt-position order across subjects.

\section{Results}

To be included in the group of subjects used for statistical analysis, a subject had to obtain at least $75 \%$ correct responses in each of the head-tilt positions on both the naming and the discrimination tasks. As noted previously, 48 subjects were accepted and 4 were rejected according to this criterion. 
For each task, the reaction times were submitted to two sets of analyses. One set was based simply on the correct response times, excluding outlier times of greater or less than 3.0 standard deviations from the mean for each cell, for each subject. Response times were rejected recursively, on the basis of new cell means, until the data set satisfied this criterion. None of the correct naming responses and $1.0 \%$ of the correct mirror-normal discrimination responses were rejected with this criterion.

The second set of analyses was based on estimates of the orientation of the subjective upright for the reference frame $(\theta)$ used by the subjects under each condition. For these analyses, a single subjective frame value was estimated for each condition such that the correlation between the actual pattern of results across orientations and the ideal pattern of contrasts expected for that particular orientation of the subjective frame of reference was maximized. The ideal contrasts were computed and fit iteratively to the response pattern over the six stimulus orientations for each subject in each condition, until the contrasts that accounted for the most variance in the response pattern were found.

In a manner similar to that described by Corballis, Nagourney, et al. (1978), the ideal contrasts were based on the assumption that the response times are a linear function of the degree to which a stimulus is orientationally misaligned with the subjective upright. For example, the ideal pattern for each orientation used in the study (i.e., $0^{\circ}, 60^{\circ}, 120^{\circ}, 180^{\circ}, 240^{\circ}$, and $300^{\circ}$ ) for a frame of reference at $0^{\circ}$ was $0,60,120,180,120$, and 60 . Likewise, the pattern for a frame of reference at $-60^{\circ}$ (as would be expected with a retinal reference frame) was 60,120 , $180,120,60$, and 0 , and, finally, the pattern for a frame of reference at $60^{\circ}$ was $60,0,60,120,180$, and 120 .

Lower and upper limits for the subjective frames to be tested were set for each head-position condition (e.g., -60 and 180 for the right-tilt condition). The contrasts that were used on the first pass of the fitting procedure corresponded to the lower limit subjective frame value. Therefore, in this example, the ideal set of contrasts for the first pass (for the lower limit, $\theta=-60$ ) was 60,120 , $180,120,60$, and 0 . A badness-of-fit value that estimated the amount of variance in the response pattern not accounted for by the ideal contrasts was derived $\left(1-r^{2}\right)$, and the fitting procedure minimized this value.

The general formula used to derive the ideal contrasts was the following:

$$
\text { Contrast Value }=|180-| \text { Stim }-\theta-180|| \text {. }
$$

In this formula, $\theta$ is the subjective frame value being tested, Stim is one of the stimulus-orientation values $(0,60,120$, 180,240 , or 300 ) used in this study, and Contrast Value is the ideal contrast that corresponds to the Stim value. This formula was used to compute the six ideal contrast values for any given subjective frame orientation.

The next pass of the frame-estimation procedure incremented the subjective frame to be tested by $2^{\circ}$. In this example, the second pass tested a subjective frame of $-60+2=-58^{\circ}$. The ideal contrasts for each of the stimulus orientations in this study for a subjective frame of $-58^{\circ}$ are $58,118,178,122,62$, and 2 . If more variance was accounted for by these ideal contrasts than the previous ones, then iterations continued in this direction until the iteration $n+1$ accounted for less variance than iteration $n$. At that point, the iteration increment was decreased from 2 to 0.01 , for example, to get a finer measure of the subjective frame, and the estimation procedure continued. The subjective frame value for which no further iterations increased the amount of variance accounted for in the actual response pattern for a design cell was taken as the best estimate of the subjective frame used by that subject under those particular conditions.

As an idealized example, a subject with head tilted $60^{\circ}$ to the right and using a perfectly retinally aligned frame would produce a response pattern with fastest responses to stimuli at $60^{\circ}$ and responses that increase linearly to stimuli increasingly rotated from $60^{\circ}$. Such a pattern of responses would be assigned a subjective upright estimate of $+60^{\circ}$ (see Appendix B, broken-line curve). On the other hand, a subject with head tilted $60^{\circ}$ to the right using a perfectly environmentally aligned frame would respond most quickly to stimuli at $0^{\circ}$ and increasingly slowly to those increasingly misaligned from $0^{\circ}$. This pattern of responses would produce a subjective upright estimate of $0^{\circ}$ (see Appendix C, broken-line curve).

If the average subjective upright estimates approximate rather than equal $0^{\circ}$ or $60^{\circ}$, there are two possible interpretations: (1) a probabilistic interpretation in which the frame is perfectly aligned with one of the two sets of directions on each trial and on average is more frequently aligned with one particular set, or (2) an interpretation in which the frame rarely is aligned perfectly with either set of directions and is instead somewhere between the two sets of directions throughout any particular trial.

\section{Naming}

Response time. For each subject, naming times for correct responses were averaged over items for each headtilt condition and each orientation. These means were submitted to an analysis of variance (ANOVA) with group (upright and left head tilt or upright and right head tilt) as a between-subjects factor and tilt (upright or tilted) and stimulus orientation $\left(0^{\circ}, 60^{\circ}, 120^{\circ}, 180^{\circ}, 240^{\circ}\right.$, or $\left.300^{\circ}\right)$ as within-subjects factors. Figure 1 shows the mean naming latency, across subjects, at each orientation for each of the three head-tilt conditions in the experiment. Response times from the head-upright conditions in the two head-tilt groups were averaged for this figure.

The time to name a disoriented object was sharply affected by the degree of disorientation $[F(5,230)=9.6$, $p<.001]$. Furthermore, the pattern of this effect depended on the group $[F(5,230)=4.8, p<.001]$. The difference between the effects of stimulus orientation in the head-upright and -tilted conditions also should have differed for the two groups and should have produced a three-way interaction between group, tilt, and orientation 


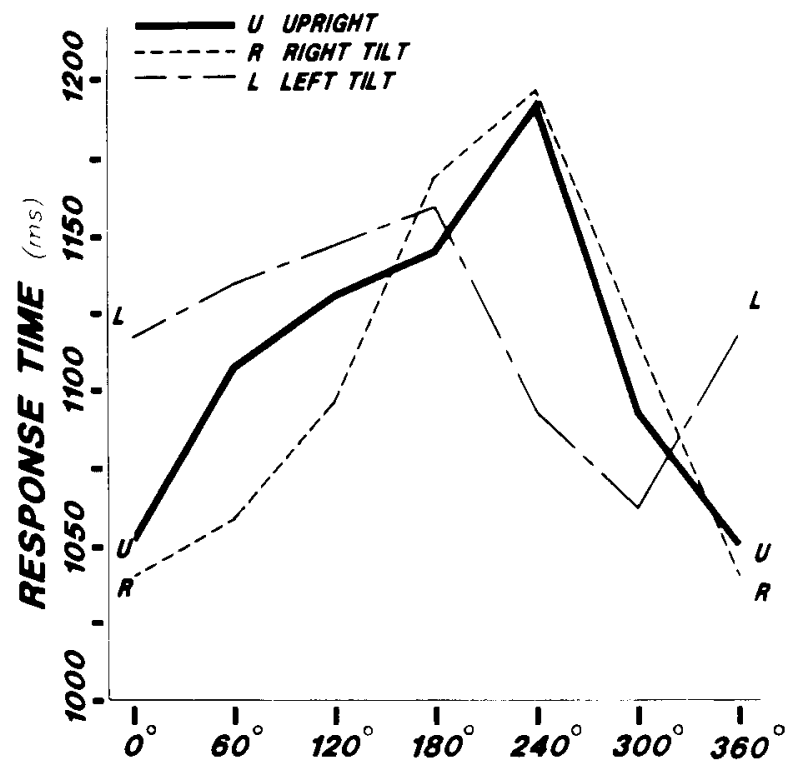

STIMULUS ORIENTATION

Figure 1. Mean response time to name all 120 objects in Experiment 1 for each stimulus orientation and head-tilt position.

if the reference frame was predominantly retinal. However, this interaction was not significant $[F(5,230)=1.3$, $p<.3]$.

Separate analyses of the data within each tilt group revealed that the effects of orientation differed when the head was tilted left relative to when the head was upright for the left-tilt group $[F(3,115)=2.5, p<.05]$. However, the effects of orientation when the head was tilted right relative to when it was upright for the right-tilt group were not different $[F(5,115)=0.4, p<.9]$. The overall interaction between group and orientation does, however, seem to have been due to different effects of orientation between the left and right head-tilt conditions $[F(5,230)=4.4, p<.001]$ because the effects of orientation were not different for the head-upright conditions for the two groups $[F(5,230)=1.2, p<.3]$.

An item analysis was performed to further investigate the effect of head-tilt position on the effects of stimulus orientation. For this analysis, the naming times for each head-tilt position (upright, left, and right) and each orientation, for each item, were averaged across subjects. As before, an outlier rejection criterion of 3.0 standard deviations from the mean for each item in each condition and orientation was implemented, which failed to reject any of the correct responses.

These means were submitted to an ANOVA in which head-tilt position and orientation were considered to be within-item factors. Since only 4 subjects responded to any one item in the head-upright position at any particular orientation and only 2 subjects responded to any one item in the head-left or -right positions at any particular orientation, there were no correct responses in one of the cells (item $\times$ head-tilt position $\times$ orientation) for 25 of the items. These 25 items were excluded, resulting in an analysis based on 95 items.

As in the subject analysis, there was a large overall effect of orientation $[F(5,470)=9.0, p<.001]$. More importantly, the overall effect of orientation depended on the head-tilt position $[F(10,940)=2.7, p<.01]$. This effect suggests that the failure to find significant effects of head position on the effects of orientation in the subject analysis may have been due to individual items or to insufficient statistical power attributable to the betweensubject design of the previous analysis. The effect of headtilt position on the effects of orientation for the 95 items in this analysis can be seen in Figure 2 . Note that excluding the 25 error-prone items particularly affected the average response times to stimuli at $120^{\circ}$ and $240^{\circ}$ in the upright condition. These results lend some support to the view that novel depictions of rotated objects are identified within a predominantly retinally aligned frame of reference.

Subjective upright. Subjective upright values were estimated from the mean correct response times to all 120 objects for each head tilt, each orientation, and each subject. These estimates were submitted to an ANOVA with group (upright and right tilt or upright and left tilt) as a between-subjects variable and tilt (upright or tilted) as a within-subjects variable.

The subjective upright was different overall for the two groups $[F(1,46)=20.2, p<.001]$. Also, the effect of head tilt depended on group $[F(1,46)=16.6, p<.001]$. Planned comparisons revealed that subjective upright estimates from the head-upright condition were different

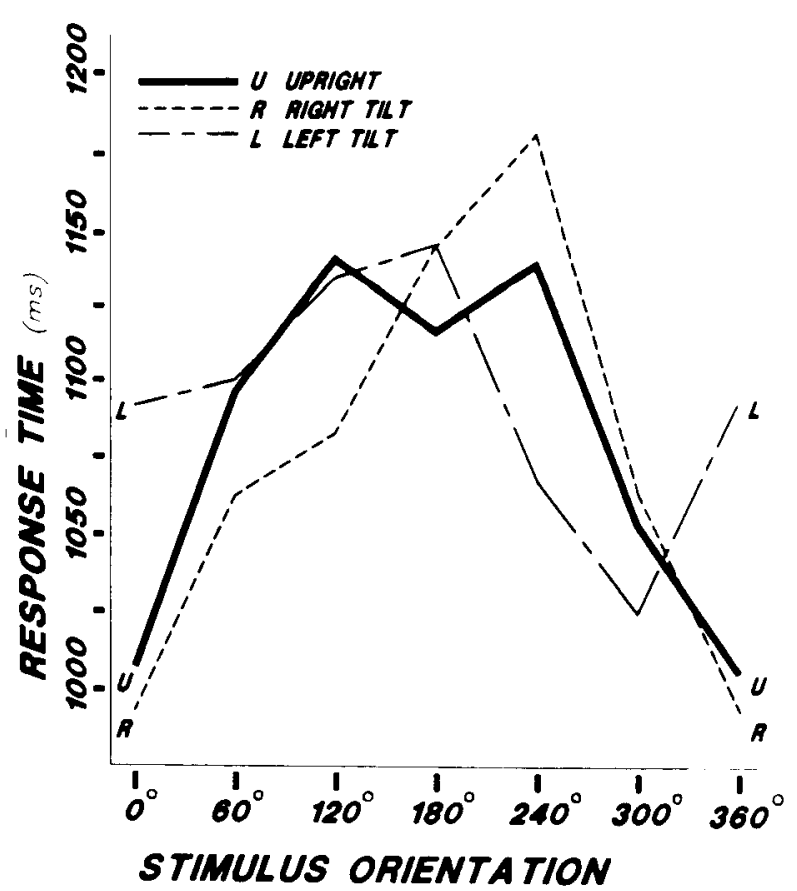

Figure 2. Mean response time to name the 95 objects included in the item analysis in Experiment 1 for each stimulus orientation and head-tilt position. 


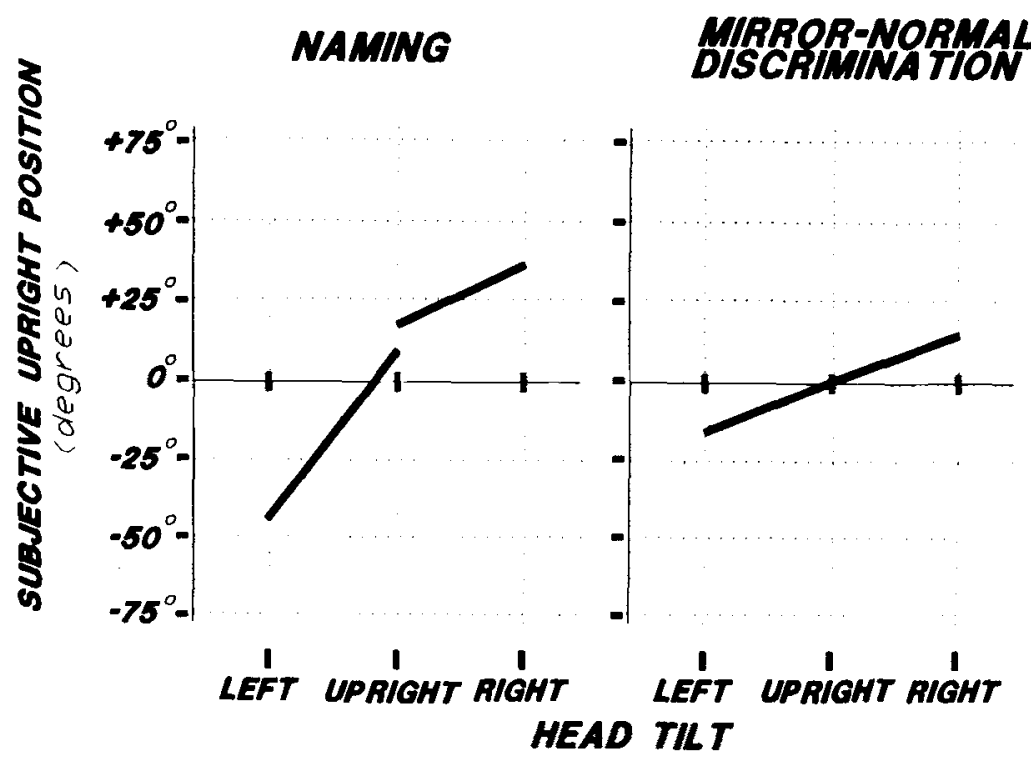

Figure 3. Mean subjective upright estimates (in degrees from the environmental vertical) in the naming task (left panel) and the mirror-normal discrimination task (right panel) in Experiment 1 for each head-tilt position.

from those from the head tilted left condition $[F(1,23)=$ $12.7, p<.01]$. Head-upright estimates also were different from head tilted right estimates $[F(1,23)=4.1$, $p<.05]$. These effects are illustrated in the left panel of Figure 3.

The average departure from the environmental upright of the frame orientation in the head-tilted conditions was $36.4^{\circ}$ in the direction of head tilt. Two other estimates of the average frame of reference across head-tilt directions were determined. A single, subjective frame orientation was derived from the average response-time pattern to all 120 objects across all subjects in each head-tilt condition (see Figure 1). In this analysis, the orientation of the average frame was $49.9^{\circ}$ in the direction of head tilt. The same estimate from the response-time pattern to the 95 objects used in the item analysis was $35.9^{\circ}$. These estimates of the orientation of the average subjective frame may reflect less individual noise than the first estimate of the average frame orientation reported above. Regardless of which estimate is used, the orientation of the subjective frame in the naming task was closer to the retinal upright than to the environmental upright, although it coincided with neither extreme.

Errors. The main dependent measures in this study were response time and subjective upright estimate; however, error rates from responses to the entire 120 -item stimulus set also were analyzed to ensure that the response-time results were not due to speed-accuracy tradeoffs. Trials in which subjects used an inappropriate name, made a hesitation utterance such as "ah," or prematurely tripped the voice key were treated as errors and discarded. ${ }^{3}$ The average percent-error rate for each stimulus orientation and head-tilt condition for each group can be found in Table 1 .
An ANOVA on the average error rates demonstrated overall effects of orientation $[F(5,230)=3.3, p<.01]$ that generally were consistent with the response-time results. No other effects were significant in this analysis. The errors in the upright condition indicate that the subjective frame was biased to the left, whereas the reactiontime data indicate a frame bias to the right. This suggestion of a speed-accuracy tradeoff could account for the skewed upright response curve in Figure 1. Except for the upright condition, the error rates did not suggest that the effect of orientation on naming time could be due to speed-accuracy tradeoffs.

\section{Mirror-Normal Discrimination}

Response time. Correct mirror-normal discrimination times were averaged over items for each head-tilt condition, orientation, and subject. These response times were analyzed using an ANOVA with both tilt (upright, left, or right) and orientation $\left(0^{\circ}, 60^{\circ}, 120^{\circ}, 180^{\circ}, 240^{\circ}\right.$, or $\left.300^{\circ}\right)$ as within-subject variables.

Figure 4 shows the mean latency for each orientation and head-tilt condition. Stimulus orientation had a large

Table 1

Mean Percent-Error Rates in the Naming Task for Each Group, Head-Tilt Position, and Stimulus Orientation in Experiment 1

\begin{tabular}{lrrrrrr}
\hline \multirow{8}{*}{$\begin{array}{l}\text { Head-Tilt } \\
\text { Position }\end{array}$} & \multicolumn{7}{c}{ Stimulus Orientation (in degrees) } \\
\cline { 2 - 6 } & 0 & 60 & 120 & 180 & 240 & 300 \\
\hline \multirow{7}{*}{ Upright/Left Group } \\
Upright & 5.4 & 9.2 & 10.0 & 11.2 & 9.6 & 8.3 \\
Tilted & 5.0 & 10.0 & 14.6 & 8.3 & 7.9 & 8.3 \\
& \multicolumn{7}{c}{ Upright/Right Group } \\
Upright & 6.7 & 11.7 & 11.7 & 15.4 & 11.7 & 9.6 \\
Tilted & 10.8 & 10.8 & 7.1 & 12.1 & 12.1 & 10.0 \\
\hline
\end{tabular}




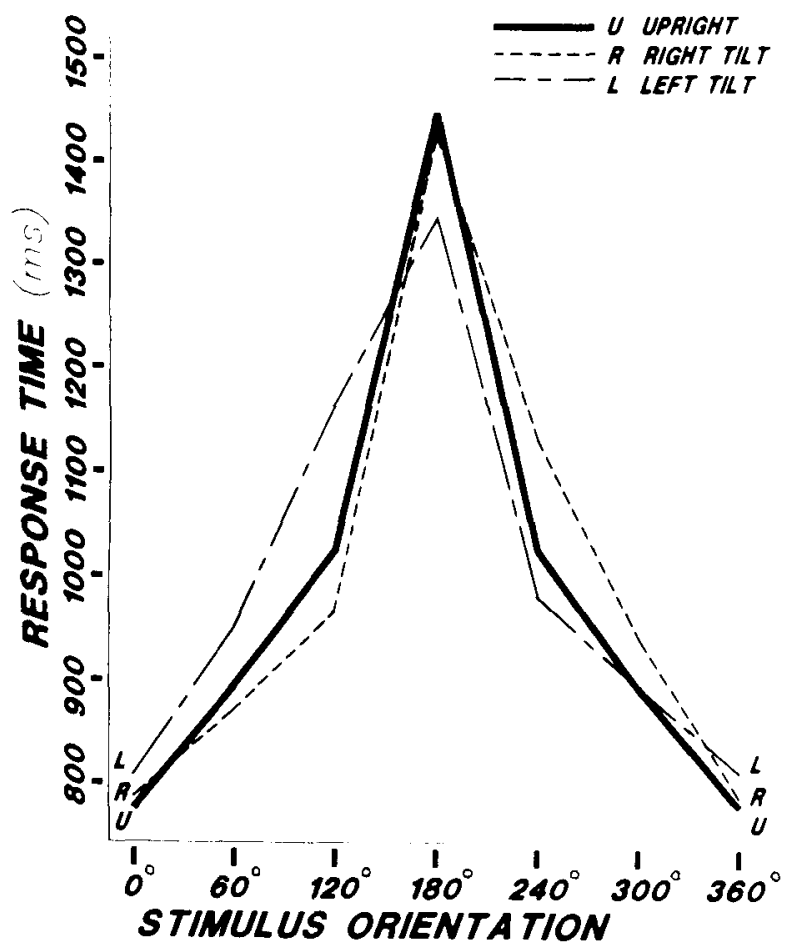

Figure 4. Mean response time to discriminate mirror-imaged from normal characters in Experiment 1 for each stimulus orientation and head-tilt position.

effect on performance $[F(5,235)=155.0, p<.001]$. In addition, this effect varied with head tilt $[F(10,470)=$ $14.0, p<.001]$. However, the response patterns in Figure 4 clearly indicate that the fastest and slowest responses occurred at $0^{\circ}$ and $180^{\circ}$ of orientation relative to the environment, respectively. This response pattern is consistent with the use of an environmentally aligned reference frame, as is suggested further by the analysis of the subjective frames presented below.

Subjective upright. Subjective upright values were estimated from the mean correct response times for each orientation, each subject, and each head tilt. The average of these estimates for each head-tilt condition, collapsed over subjects, can be seen in the right panel of Figure 3. An ANOVA on these data considered head tilt (upright, left, or right) as a within-subjects variable. This analysis showed that subjective upright depended on head tilt $[F(2,94)=39.0, p<.001]$, which agrees with the analysis based on the mean response times.

The average departure from the environmental upright of the frame orientation in the head-tilted conditions was $16.8^{\circ}$ in the direction of head tilt. Alternatively, deriving a single, average subjective frame orientation from the average response-time pattern across all subjects in each head-tilt condition (see Figure 4) resulted in an estimate of $14.1^{\circ}$ in the direction of head tilt. This single estimate of the orientation of the average subjective frame in the mirror-normal discrimination task and the estimate based on the average of the frame estimates across all subjects $\left(16.8^{\circ}\right)$ indicate that the subjective frame was closer to the environmental upright than to the retinal upright.

Errors. The percent-error rates for each head-tilt condition and orientation were analyzed to determine whether the mirror-normal discrimination results found with the reaction-time analysis could have been due to speedaccuracy tradeoffs. The percent-error rate at each orientation and head tilt is shown in Table 2.

An analysis of these error rates revealed an effect of stimulus orientation $[F(5,235)=38.6, p<.001]$ that depended on the head-tilt condition $[F(10,470)=4.2$, $p<.001]$. These effects were consistent with those demonstrated with the response-time analyses and failed to indicate that the effects shown with the response-time measure were due to speed-accuracy tradeoffs.

\section{Naming and Mirror-Normal Discrimination}

Mean subjective upright estimates from both the naming and the mirror-normal discrimination tasks were analyzed together in a group (upright and right tilt or upright and left tilt) $\times$ task (naming or mirror-normal discrimination) $\times$ tilt (upright or tilted) design, with group as a between-subjects variable and the other factors as withinsubjects variables. Only subjective upright estimates from the discrimination task from the two tilt positions common to both tasks for a subject were used in this analysis. Figure 3 graphically compares the mean subjective upright estimates for each task and head-tilt condition.

Of primary interest was the finding that the orientation of the subjective frame was affected more by head tilt in the naming task than in the discrimination task $[F(1,46)=5.0, p<.05]$. The average subjective upright estimates in the head-tilt conditions for the two tasks corroborated this effect (naming task average frame estimate $=36.4^{\circ}$ in the direction of tilt; mirror-normal discrimination task average frame estimate $=14.8^{\circ}$ in the direction of head tilt).

Concomitant with this pattern of results was a main effect of group membership (upright and left tilt or upright and right tilt) $[F(1,46)=33.2, p<.001]$, which was greater for the naming than for the discrimination task $[F(1,46)=$ $7.4, p<.011$. The effect of head tilt collapsed across tasks was also different for the two groups $[F(1,46)=$ $31.3, p<.001]$. These results are consistent with the finding that while both naming and mirror-normal discriminations are affected by head tilt, the naming task is influenced more strongly by this variable.

Table 2

Mean Percent-Error Rates in the Mirror-Normal Discrimination Task for Each Head-Tilt Position and Stimulus Orientation in Experiment 1

\begin{tabular}{lcccccc}
\hline \multirow{2}{*}{$\begin{array}{l}\text { Head-Tilt } \\
\text { Position }\end{array}$} & 0 & 60 & 120 & 180 & 240 & 300 \\
\cline { 2 - 7 } & 0 & Stimulus Orientation (in degrees) & \\
\hline Left & 2.7 & 3.1 & 6.8 & 12.7 & 2.1 & 2.7 \\
Upright & 2.5 & 3.1 & 4.6 & 12.2 & 4.1 & 2.4 \\
Right & 2.6 & 2.1 & 4.1 & 11.7 & 8.1 & 2.6 \\
\hline
\end{tabular}




\section{Discussion}

Consistent with previous results, stimulus orientation affected object-naming time and accuracy in a roughly linear fashion (Jolicoeur, 1985, 1988; Jolicoeur \& Milliken, 1989; Jolicoeur, Snow, \& Murray, 1987; Maki, 1986; Shwartz, 1981). The new finding was that the orientation effect in naming occurred within a reference frame whose orientation was strongly affected by the direction of head tilt and which, on average, was somewhat closer to the retinal vertical (by $24^{\circ}$ ) than to the environmental vertical (by $36^{\circ}$ ). This estimate of the subjective frame suggests a compromise between the two directional systems considered that is in favor of the retinal system. These results contrast with those from mirror-normal discriminations in which the reference frame, although also affected by head tilt, remained more closely aligned with the environmental vertical.

Given that the stimuli in the present and upcoming experiments were always presented within an environmentally upright rectangular border that could serve as a strong cue to the environmental directions (Corballis, Nagourney, et al., 1978; Rock, 1956) and that there was no instructional bias to use a retinal frame (Attneave \& Olson, 1967; Attneave \& Reid, 1968; Corballis, Nagourney, et al., 1978; Rock, 1973; Rock \& Heimer, 1957), we find all findings consistent with the use of a more retinally aligned reference frame particularly impressive.

Corballis, Anuza, and Blake (1978) have reported that effects of stimulus orientation on letter naming occur within a frame aligned predominantly with retinal directions but only when the stimuli are displayed within a circular surround. The present results generalize this finding to object naming.

The effect of head tilt on mirror-normal discrimination response patterns across stimulus orientations is consistent with previously reported findings (Corballis, Nagourney, et al., 1978). However, both the present results and those of Corballis, Nagourney, et al. showed that the fastest and slowest responses invariably occurred to stimuli oriented $0^{\circ}$ and $180^{\circ}$ relative to the environment, respectively. The effect of head tilt, then, was predominantly upon stimuli oriented at oblique angles. This contrasts with the results from the naming task, which showed that stimuli at all orientations were affected by head tilt. Although all dependent measures indicated overall significant effects of head tilt on mirror-normal discriminations, the estimates of the average orientation of the subjective frame of reference were much closer to the environmental upright than to the retinal upright.

Finally, the results of the analysis that compared subjective upright estimates for both tasks found larger effects of head tilt on naming than on mirror-normal discriminations. Together with the average subjective upright scores themselves, these results suggest that different spatial referents are used under different perceptual conditions. This conclusion contrasts with Rock's $(1973,1974)$ view that rotated forms are perceived, in general, within a frame of reference that is aligned predominantly with the environment, although he has suggested that certain stimulus attributes such as low familiarity or high complexity may contribute to the use of a more retinally aligned frame (Rock, 1973, 1974; Rock \& DiVita, 1987). In the next experiment, we investigated which attributes of our experimental paradigm determine the spatial direction system used: those of the stimulus set or those of the task.

\section{EXPERIMENT 2}

The purpose of Experiment 2 was to replicate and extend the main finding of Experiment 1: Naming rotated objects uses a spatial reference frame that is more retinally aligned than that used for mirror-normal discriminations of rotated letters and digits. In this experiment, we examined whether the use of different spatial direction systems depends upon the task (naming vs. mirror-normal discriminations) or upon the stimuli (novel, complex objects vs. familiar, relatively simple letters and digits).

On a stimulus account, novel, complex objects that initially are interpreted within a retinally aligned frame may not have been successfully reinterpreted into an environmentally aligned frame in the naming task because these items exceeded a processing-speed deadline (Rock, 1973, p. 128) or exceeded some limits of visual capacity (Rock, 1986, p. 33-24). In this view, regardless of the task, rotated, novel, complex objects should be interpreted within a retinal frame of reference. Conversely, on a task account, mirror-normal discriminations require reference to the directions left and right (Corballis \& Beale, 1976; Hinton \& Parsons, 1981), which may be coded with respect to environmental directions (Corballis, 1988; Corballis, Nagourney, et al., 1978). This reference to environmental directions may not be necessary in object naming.

To examine which of these is the better account, a subset of the naming objects (those in lateral to three-quarter views) were discriminated on the basis of whether their front would face to the left or the right when they were imagined to be upright. This task is similar to the mirrornormal discrimination task, and it allows the use of the stimuli used in the naming task. Jolicoeur $(1985,1988)$ has shown that, like mirror-normal discriminations, response times for discriminating left-right reflections peak at $180^{\circ}$ of stimulus rotation, and that the effects of rotation are, in general, resistant to practice. By using the same stimuli for both naming and left-right reflection discriminations, any differential alignment of the spatial reference frame across the two tasks can be attributed to the tasks and not the stimuli.

\section{Method}

\section{Subjects}

Twenty-one University of Waterloo undergraduates with normal or corrected-to-normal vision participated in the experiment for pay. The data from 18 of these subjects ( 9 males) were used in the final analyses. Three subjects were excluded because they failed to reach the criterion described in the Results section. All subjects were na- 
tive speakers of English. No subject participated in any of the other experiments reported here.

\section{Apparatus and Stimuli}

The experimental set-up was as described in Experiment 1. The same 120 objects used in Experiment 1 served as stimuli for the naming task in this experiment. As well, 72 of these items that had distinct left and right orientations (a front and a back in view) were used in the left-right discrimination task (see Appendix A). As before, the stimuli were rotated frontally through six orientations.

\section{Procedure}

Naming. Naming trials always preceded left-right discriminations to avoid diminished effects of orientation on naming that occur with repeated exposure to the naming items (Jolicoeur, 1985, 1988; Jolicoeur \& Milliken, 1989; Jolicoeur et al., 1987; Maki, 1986). The naming-trial procedure was as outlined in Experiment 1, with the following exceptions. Each subject named one set of $120 \mathrm{ob}-$ jects with the head upright, the head tilted left, or the head tilted right. Twenty objects were named at each orientation. Also, 16 practice trials using objects not seen during experimental trials were presented with the head in the same position as the upcoming experimental trials.

Six sets of 120 objects each were compiled such that every object appeared once in each set in a different orientation. As before, equal numbers of objects that are named slowly and quickly when averaged across all orientations were presented in each orientation in each set. No more than four consecutive trials with the same orientation occurred in a set, and the order of presentation of the sets and head-tilt position across subjects was determined with a Latin square. Across all subjects, each set was shown in each headtilt position once. Half of the subjects in each head-tilt condition were male.

Left-right reflection discrimination. Following the naming trials, 72 left-right discrimination trials were performed under each of three conditions of head tilt. The subjects responded by pressing the right-hand key to indicate a "right-pointing" judgment and the left-hand key to indicate a "left-pointing" judgment. The fixation dot and stimulus displays were presented in the same manner as previously described for the mirror-normal discriminations. The last 16 trials from each experimental set of 72 objects were repeated as practice trials before each head-tilt-condition session with the head in the position of the upcoming trials.

The same six sets used in the naming trials were used in the left-right discrimination trials, with the exception of the last 48 objects in each set, which were excluded because they did not have distinct left and right orientations. A different set was shown at each head-tilt position, which resulted in 12 objects shown in each orientation at each head-tilt position. No subject ever saw the same object in the same orientation more than once. No more than four consecutive trials contained objects of the same orientation or left-right response. Across all subjects, all objects were shown in all orientations equally often, and all objects pointed either to the left or to the right equally often. Each object pointed to the left for three orientations $\left(60^{\circ}, 180^{\circ}\right.$, and $300^{\circ}$ or $0^{\circ}, 120^{\circ}$, and $240^{\circ}$ ) and to the right for the other three orientations. Objects that pointed in one direction in the slide mount, but were required to point in the other direction were left-right reversed in the slide trays. A Latin square determined the three head-tilt condition orders across subjects, and across all subjects, each set was shown once in each head-tilt position.

\section{Results}

The criterion for inclusion of subjects in the data analysis was $75 \%$ correct responses in the naming task. Eigh- teen subjects were accepted according to this criterion and 3 were rejected.

As before, response time and subjective upright estimate were the principal dependent measures, and errors were again analyzed to determine if speed-accuracy tradeoffs had occurred. The outlier rejection criterion for response times again was 3.0 standard deviations away from the mean for each cell for each subject, with recursive rejections based on the new cell mean until the criterion was satisfied. Of the correct responses, $1.5 \%$ were outliers in the naming task and $0.2 \%$ were outliers in the left-right discrimination task.

\section{Naming}

Response time. The mean correct naming latencies were analyzed with head-tilt position (upright, left, or right) as a between-subjects factor and orientation $\left(0^{\circ}, 60^{\circ}\right.$, $120^{\circ}, 180^{\circ}, 240^{\circ}$, or $300^{\circ}$ ) as a within-subjects factor.

The mean response time at each orientation for each head-tilt condition is shown in Figure 5. Naming latencies were averaged over objects for each head-tilt position, each orientation, and each subject. ${ }^{4}$ An ANOVA on these means indicated that overall stimulus orientation affected performance $[F(5,75)=4.3, p<.01]$ and that this effect depended on head tilt $[F(10,75)=2.5, p<.01]$. In this experiment, both the patterns of naming-response latencies shown in Figure 5 and the analysis of these data suggest a substantial influence of head tilt, and the size of this effect, in turn, strongly supports the use of a predominantly retinally aligned frame of reference.

In addition, an item analysis of the response times was performed and found to confirm all of the results of the subject analysis.

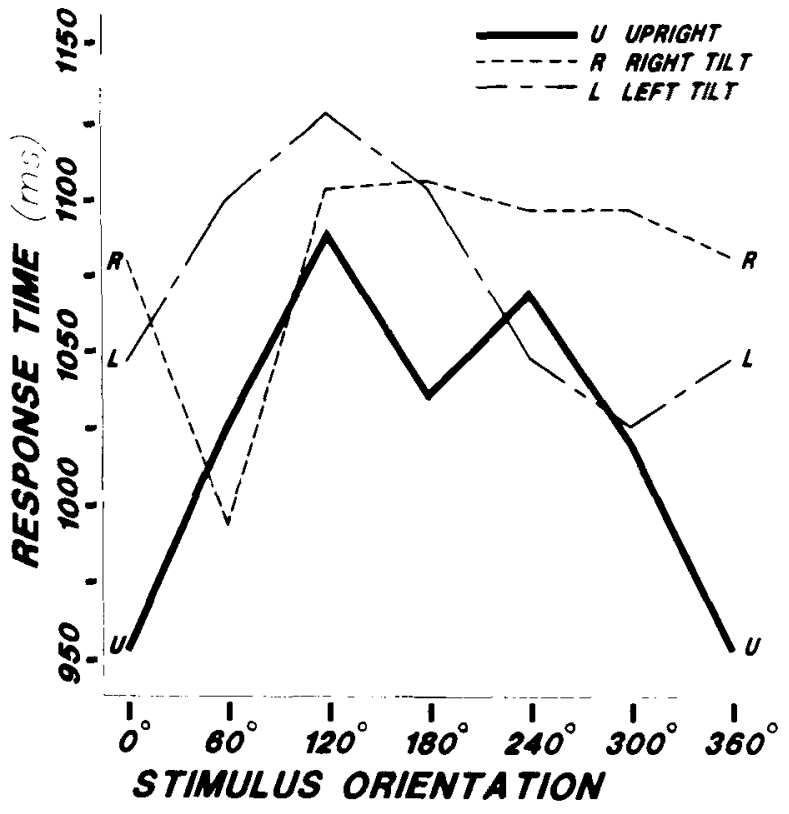

Figure 5. Mean response time to name objects in Experiment 2 for each stimulus orientation and head-tilt position. 


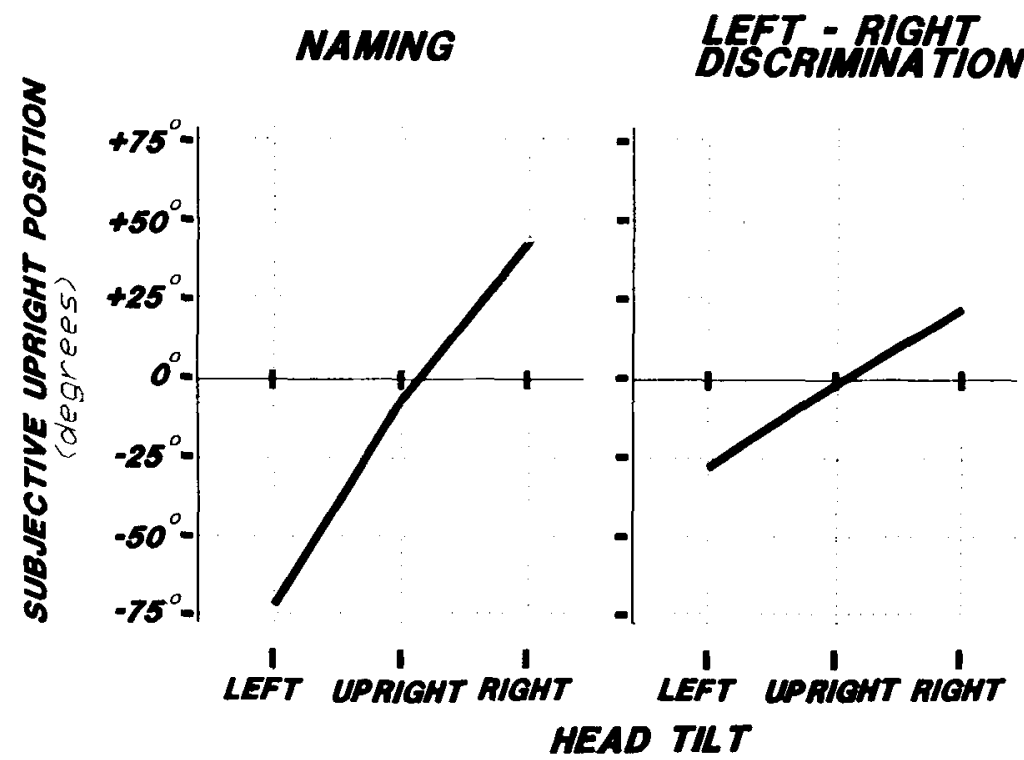

Figure 6. Mean subjective upright estimates (in degrees from the environmental vertical) from the naming task (left panel) and the left-right reflection task (right panel) in Experiment 2 for each head-tilt position.

Subjective upright. The average subjective upright estimates under each head-tilt condition are plotted in the left panel of Figure 6. The average subjective upright was different for each head-tilt position $[F(2,15)=21.0$, $p<.001]$. This result agrees with the findings from Experiment 1 .

The average orientation of the subjective frame in the head-tilt conditions from the environmental upright was $59.1^{\circ}$ in the direction of head tilt. The orientation of the subjective frame based on the average response-time pattern in each head-tilt condition across all subjects (see Figure 5) was $56.1^{\circ}$ in the direction of head tilt. Both estimates of the orientation of the subjective frame in the naming task were very much closer to the retinal upright than to the environmental upright.

In addition, the average subjective upright estimates from the naming task in Experiment 1 and the naming task in Experiment 2 were analyzed together. The effect of head-tilt position was not different for the two naming experiments $[F(2,60)=1.6, p<.2]$.

Errors. Error rates were analyzed to detect any evidence of speed-accuracy tradeoffs using the model previously described in the response-time section. The average percent-error rates at each orientation and head tilt are shown in Table 3. Analyses of the error rates failed to reveal any systematic effects of either head tilt or orientation. These results do not suggest speed-accuracy tradeoffs.

\section{Left-Right Reflection Discrimination}

Response time. Figure 7 shows the average correct left-right reflection-discrimination times for each condition. The correct response latencies were averaged over objects for each head-tilt condition, orientation, and sub- ject, and were analyzed with head-tilt position (upright, left, or right $)$ and orientation $\left(0^{\circ}, 60^{\circ}, 120^{\circ}, 180^{\circ}, 240^{\circ}\right.$, or $\left.300^{\circ}\right)$ as within-subject variables.

Once again, there was an overall effect of stimulus orientation $[F(5,85)=45.4, p<.001]$ that depended on head-tilt position $[F(10,170)=5.4, p<.001]$. Like the previous analyses of the mirror-normal discrimination data, this influence of head-tilt position on the effect of orientation appears to stem from objects at oblique orientations, because the slowest and fastest latencies were always produced by orientations of $0^{\circ}$ and $180^{\circ}$, respectively. The pattern of responses suggests that the subjective upright was more closely aligned with environmental directions than with retinal directions.

Subjective upright. An ANOVA on the subjective upright estimates was performed with tilt (upright, left, or right) as a within-subjects variable. The overall subjective upright was affected by head tilt $[F(2,34)=39.5$, $p<.001]$, as is shown in the right panel of Figure 6 . This effect is consistent with that demonstrated by the response-time analyses.

The average departure of the subjective frame from the environmental upright in the head-tilt conditions was $22.9^{\circ}$ in the direction of head tilt. When a single subjective frame orientation was estimated from the average response-

Table 3

Mean Percent-Error Rates in the Naming Task for Each Head-Tilt Position and Stimulus Orientation in Experiment 2

\begin{tabular}{|c|c|c|c|c|c|c|}
\hline \multirow{2}{*}{$\begin{array}{c}\text { Head-Tilt } \\
\text { Position }\end{array}$} & \multicolumn{6}{|c|}{ Stimulus Orientation (in degrees) } \\
\hline & 0 & 60 & 120 & 180 & 240 & 300 \\
\hline Left & 10.0 & 10.8 & 10.0 & 9.2 & 10.0 & 9.2 \\
\hline Upright & 6.7 & 10.0 & 15.8 & 11.7 & 12.5 & 10.0 \\
\hline Right & 5.8 & 10.8 & 7.5 & 10.0 & 13.3 & 11.7 \\
\hline
\end{tabular}




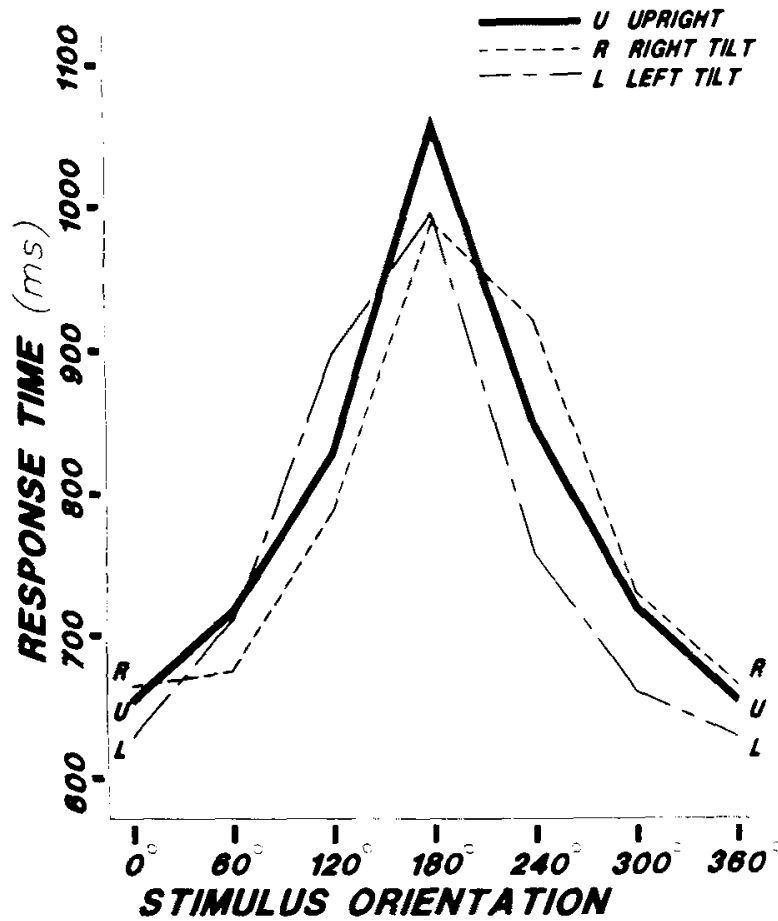

Figure 7. Mean response time to discriminate left-pointing from right-pointing objects in Experiment 2 for each stimulus orientation and head-tilt position.

time pattern in each head-tilt condition across all subjects (see Figure 7), the estimate was $19.6^{\circ}$ in the direction of head tilt. Both of these average frame estimates indicate that in the left-right reflection-discrimination task, the orientation of the subjective frame was closer to the environmental upright than to the retinal upright.

Mean subjective upright estimates from the left-right reflection and mirror-normal discrimination (Experiment 1) tasks were analyzed together, with task (left-right reflection or mirror-normal) as a between-subjects variable and tilt position (upright, left, or right) as a withinsubjects variable. Compare the subjective frame estimates from the right panel of Figure 3 with those from the right panel of Figure 6. The ANOVA showed that the effects of head-tilt position were not different for the two discrimination tasks $[F(2,128)=1.8, p<.2]$, suggesting that performance of both tasks relied on the same spatial referents.

Errors. The average percent-error rate at each orientation and head tilt is shown in Table 4. The mean errorrate analysis showed only an overall effect of stimulus orientation that was consistent with that found with the latency analysis $[F(5,85)=6.2, p<.001]$. These findings do not support the view that the effects found in the latency analysis were due to speed-accuracy tradeoffs.

\section{Naming and Left-Right Reflection Discrimination}

The mean subjective upright estimates from both the naming and the left-right reflection-discrimination tasks were analyzed together, with tilt position (upright, left, or right) as a between-subjects variable and task (naming or left-right reflection discriminations) as a withinsubjects variable. Only subjective upright estimates from the discrimination responses to the single head-tilt position common to both tasks for a subject were used in this analysis. Figure 6 compares the mean subjective upright estimates for each task and head-tilt condition.

An ANOVA of these data revealed that head-tilt position had an overall effect $[F(2,15)=39.9, p<.001]$. Of particular interest was the finding that head-tilt position had a greater effect on the orientation of the subjective upright in the naming task than in the left-right reflection task $[F(2,15)=4.2, p<.05]$. This result reflects the different effects of head-tilt position on the two tasks that are apparent when the patterns of response times in Figures 5 and 7 are contrasted. In addition, the average subjective upright estimate for the naming task was $59.1^{\circ}$ in the direction of head tilt, suggesting a very strong retinal effect. In contrast, the estimate for the left-right reflection task (based on only the single head-tilt condition common to a subject in the naming and left-right discriminations) was $24.4^{\circ}$. These results demonstrate convincingly that the naming task was performed within a reference frame aligned more closely with retinal directions than was the left-right reflection task.

\section{Discussion}

Different frames of reference (environmental or retinal) are used in different tasks. Since the same stimuli were used in both the naming and the left-right reflection tasks, the differences in the subjective frames across tasks cannot be due to differences in the nature of the stimuli, as could have been the case in Experiment 1.

As in Experiment 1, naming latencies were affected by stimulus orientation, and this effect varied with head-tilt position. The average orientation of the subjective frame was very close to the orientation of the retinal meridian $\left(59.1^{\circ}\right)$ in the head-tilt conditions, and this estimate was not statistically different from that found in Experiment 1 $\left(36.4^{\circ}\right)$. Together, these findings strongly suggest that a reference frame predominantly aligned with retinal directions was used in the naming task.

The left-right reflection task also showed a main effect of stimulus orientation that depended on head tilt. However, the response-time pattern indicated that the effect of head tilt was confined to oblique orientations. Head tilt must have had little effect on stimuli at $0^{\circ}$ and $180^{\circ}$ of disorientation because stimuli at these orientations con-

Table 4

Mean Percent-Error Rates

in the Left-Right Reflection-Discrimination Task for Each

Head-Tilt Position and Stimulus Orientation in Experiment 2

\begin{tabular}{|c|c|c|c|c|c|c|}
\hline \multirow{2}{*}{$\begin{array}{c}\text { Head-Tilt } \\
\text { Position }\end{array}$} & \multicolumn{6}{|c|}{ Stimulus Orientation (in degrees) } \\
\hline & 0 & 60 & 120 & 180 & 240 & 300 \\
\hline Left & 0.9 & 0.9 & 4.0 & 4.9 & 3.1 & \\
\hline Upright & 1.8 & 0.4 & 0.9 & 6.7 & 4.4 & \\
\hline Right & 0.9 & 0.4 & 1.8 & 3.5 & 2.2 & 2.7 \\
\hline
\end{tabular}


Table 5

Mean Subjective Reference-Frame Estimates for Normal and MirrorImaged Characters in Experiment 1 for Each Head-Tilt Position

\begin{tabular}{lccc}
\hline \multirow{2}{*}{$\begin{array}{c}\text { Character } \\
\text { Format }\end{array}$} & Left & Upright & Right \\
\cline { 2 - 4 } & -24.1 & -4.9 & 16.7 \\
Normal & -23.6 & 5.0 & 16.8 \\
\hline
\end{tabular}

sistently showed the fastest and slowest responses, respectively. This contrasts with the pattern of response times in the naming task, which changed with head tilt for stimuli at all orientations.

The fact that the same average subjective upright estimates were found for the two discrimination tasks (leftright reflection and mirror-normal), despite the fact that very different stimuli were used in each task, further supports the view that task determined which reference frame was used. The discrimination tasks have very similar response-latency characteristics (compare Figures 4 and 7) and so probably reflect the same underlying orientationsensitive mechanisms.

It is possible that differences in the frame of reference used in the naming and left-right reflection tasks were due to the fact that the stimuli were repeated in both of the left-right reflection tasks and were not repeated in the naming tasks. The results from the naming tasks could therefore be explained if low stimulus familiarity contributes to the use of a more retinally aligned frame of reference. However, two lines of evidence argue against this possibility. First, if novel stimuli are interpreted within more retinally aligned frames and increasingly rely upon more environmentally aligned frames with stimulus exposure, then we would expect to see evidence of this effect across the three blocks of presentations of the objects for left-right reflection discrimination. The average subjective frame estimates (in degrees in the direction of head tilt) from the head-tilted conditions for each block do not support this prediction: Block $1=23.3$, Block $2=$ 19.1, Block $3=26.1$.

Second, if novel stimuli are interpreted within more retinally aligned frames than are familiar stimuli, then we would expect that mirror-imaged characters were interpreted within a more retinally aligned frame than normal characters in the mirror-normal discrimination task of Experiment 1. An ANOVA on the subjective frame estimates with head-tilt position (upright, left, or right) and format (normal or mirror-imaged) as within-subject variables did not support this prediction $[F(2,94)=1.4, p<.3]$. This is apparent in Table 5.

Taken together, our results do not support the notion that novel stimuli are perceived within a more retinally aligned frame while familiar stimuli are perceived within a more environmentally aligned frame. Experiment 3 further tests this notion.

\section{EXPERIMENT 3}

Experiment 2 demonstrated that naming rotated objects depends upon a more retinally aligned frame of reference than does discriminating a left-right reflection. However, stimulus familiarity and task were confounded in that experiment. Therefore, it is possible that the average subjective reference frame was different for the two tasks because of different degrees of familiarity with the stimuli rather than differences in the nature of the tasks.

Although the frame of reference did not become more environmentally aligned with exposure to the stimulus set over three blocks of left-right reflection discriminations in Experiment 2, it is possible that the greatest effect of stimulus familiarity occurs between the first and second blocks. The left-right reflection task in Experiment 2 did not have a condition in which the stimuli had never been seen before because the stimuli were presented in the naming task before the left-right reflection task.

In order to further test the notion that regardless of the task, novel stimuli are perceived within a frame that is predominantly retinally aligned, novel object drawings were presented for left-right reflection discrimination. If novel objects are discriminated on the basis of left-right reflection within a more environmentally aligned frame of reference than when they are named, this effect would strongly indicate that the task, rather than stimulus familiarity, determines the orientation of the frame of reference.

\section{Method}

\section{Subjects}

Twelve University of Waterloo undergraduates participated in this experiment for pay. Six of these subjects were female. All subjects had normal or corrected-to-normal vision and no subject participated in any other experiment reported in this paper.

\section{Apparatus, Stimuli, and Procedure}

The same experimental set-up, stimuli, and procedure used in the left-right reflection task in Experiment 2 were used, with the following exceptions. Half of the subjects (half again of whom were female) were presented the stimuli in the head-tilt-position order left, right, and upright, whereas the other half of the subjects were shown the stimuli in the head-tilt-position order right, left, and upright. Across all subjects, each of the six-item sets were shown in each of the three head-tilt positions (left, right, and upright) twice.

\section{Results}

All subjects obtained accuracy rates of at least $75 \%$ in each of the three head-tilt positions. The outlier rejection criterion for response times that was used in Experiment 2 resulted in the rejection of $0.2 \%$ of the correct responses from the current experiment.

\section{Response Times}

The mean correct response times were analyzed with order of head-tilt position (left, right, and upright or right, left, and upright) as a between-subjects variable and headtilt position (left, right, or upright) and orientation $\left(0^{\circ}, 60^{\circ}\right.$, $120^{\circ}, 180^{\circ}, 240^{\circ}$, or $300^{\circ}$ ) as within-subject variables.

The mean correct response time at each head-tilt position and for each stimulus orientation collapsed across head-tilt position order is shown in Figure 8. As usual, orientation had a large overall effect $[F(5,50)=38.6$, $p<.001]$. As was shown in Experiment 2, this effect de- 
pended on head-tilt position $[F(10,100)=6.1, p<.001]$. Unlike Experiment 2, there was also a main effect of headtilt position $[F(2,20)=2.6, p<.001]$. Planned comparisons revealed that this effect was due to overall latency differences between the upright and the tilt conditions. The overall upright latency was faster than the overall leftposition latency $[F(1,11)=13.3, p<.01]$. Similarly, the overall upright latency was faster than the overall right-position latency $[F(1,11)=9.1, p<.01]$. However, the overall left-position latency was not different from the overall right-position latency $[F(1,11)<1]$. These effects likely represent a practice effect, because the upright position was always presented last.

\section{Subjective Upright}

An ANOVA on the absolute values of the subjective upright estimates from the two head-tilt positions was performed with block (first and second) as a within-subjects variable. Use of the absolute values of the estimates allowed an analysis that included estimates from the left head-tilt and the right head-tilt conditions, collapsing across direction of head tilt. The average of the absolute value of the frame in degrees was 26.8 for the first block and 23.5 for the second block. This difference was not significant $[F(1,11)<1]$. Thus, the frame of reference was not altered by increasing familiarity with the stimulus set between the first and second presentation blocks.

Of particular importance were the results from another analysis in which the left and right head-tilt frame estimates from the naming task in Experiment 2 were compared with the left and right head-tilt estimates from the

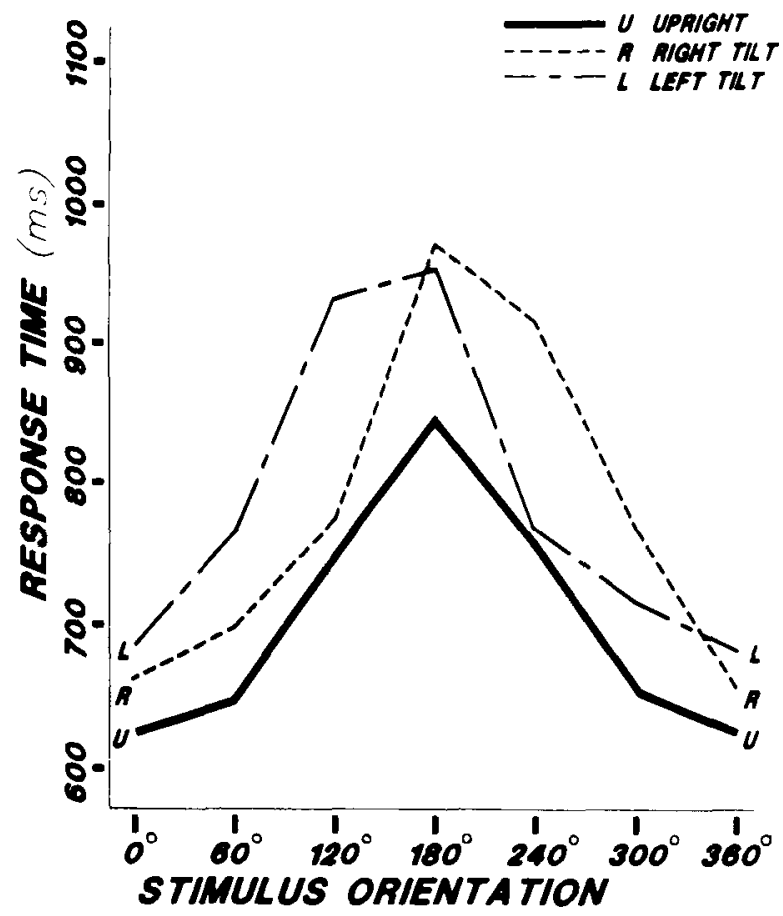

Figure 8. Mean response time to discriminate left-pointing from right-pointing objects in Experiment 3 for each stimulus orientation and head-tilt position.
Table 6

Mean Percent-Error Rates in the Left-Right ReflectionDiscrimination Task for Each Head-Tilt Position Order, Each Head-Tilt Position, and Each Stimulus Orientation in Experiment 3

\begin{tabular}{|c|c|c|c|c|c|c|}
\hline \multirow{2}{*}{$\begin{array}{l}\text { Head-Tilt } \\
\text { Position }\end{array}$} & \multicolumn{6}{|c|}{ Stimulus Orientation (in degrees) } \\
\hline & 0 & 60 & 120 & 180 & 240 & 300 \\
\hline \multicolumn{7}{|c|}{ Left, Right, Upright Order } \\
\hline Left & 1.4 & 0.0 & 4.2 & 1.4 & 2.8 & \\
\hline Upright & 1.4 & 1.4 & 1.4 & 2.8 & 0.0 & \\
\hline Right & 1.4 & 0.0 & 0.0 & 1.4 & 1.4 & \\
\hline \multicolumn{7}{|c|}{ Right, Left, Upright Order } \\
\hline Left & 0.0 & 1.4 & 6.9 & 6.9 & 1.4 & 2.8 \\
\hline Upright & 2.8 & 1.4 & 4.2 & 6.9 & 5.5 & 2.8 \\
\hline Right & 1.4 & 1.4 & 4.1 & 6.9 & 4.2 & 2.8 \\
\hline
\end{tabular}

first block of left-right reflection discriminations in the current experiment. In this analysis, task (naming or leftright reflection discrimination) and head-tilt position (left or right) were between-subject variables. The effect of head-tilt position on the frames from the naming task was greater than the effect on the frames from the left-right reflection task $[F(1,20)=13.2, p<.01]$. In this analysis, the average frame of reference in degrees in the direction of head tilt was 59.1 for naming in Experiment 2 and 25.0 for the left-right reflection-discrimination task.

Overall, the average estimate of the frame of reference in the current experiment, based on the average frame estimates in the two tilt conditions across subjects and blocks, was $22.2^{\circ}$ in the direction of head tilt. This estimate is very similar to the corresponding estimate of $22.9^{\circ}$ computed in Experiment 2. When a single estimate of the average frame of reference in the current experiment was based on the average response time collapsed across subjects and blocks (see Figure 8), a very similar estimate of $23.8^{\circ}$ in the direction of head tilt was obtained.

Overall, these results clearly indicate that left-right discriminations are performed within a frame of reference that is more closely aligned with environmental directions, regardless of the familiarity of the stimulus set.

\section{Errors}

The average percent-error rate for each head-tilt position order, stimulus orientation, and head-tilt position is shown in Table 6. The mean error-rate analysis demonstrated an overall effect of orientation that was consistent with that demonstrated in the response-time analysis $[F(5,50)=3.7, p<.01]$. As well, head-tilt-position order had an overall effect that showed that the subjects who received the head-tilt position order of left, right, and upright produced fewer errors (mean $=1.5 \%$ ) than did the subjects who received the head-tilt position order of right, left, and upright $($ mean $=3.5 \%)[F(1,10)=7.6, p<.05]$. Since this effect is based on very few data points, it is unclear what this difference reflects, and we do not attempt to interpret it.

\section{Discussion}

Experiments 2 and 3 demonstrate that when stimulus familiarity is held constant, the frame of reference for 
left-right reflection discrimination is more environmentally aligned than the frame for naming. The average subjective frame estimate when novel objects were named in Experiment 2 was $59.1^{\circ}$ in the direction of head tilt, while the same estimate when novel objects were discriminated on the basis of left-right reflection was $25.0^{\circ}$ in the direction of head tilt.

In addition, this effect agrees with the results of Experiment 2 that showed that the average subjective frame estimate did not change over three blocks of left-right reflection discriminations. The results of the current experiment indicate that there is no large shift in the frame of reference from the first presentation block of the stimulus set (average frame $=25.0^{\circ}$ in the direction of head tilt) to the second block (average frame $=23.3^{\circ}$ in the direction of head tilt from Experiment 2). This possibility could not be ruled out in Experiment 2. Moreover, the current results support the conclusion drawn from the results of Experiment 1 that showed that the frame-ofreference estimates were not different for mirror-imaged and normal discriminations.

No support for the notion that novel stimuli are perceived within more retinally aligned frames of reference than are familiar stimuli has been found. Thus, the differences in the orientation of the subjective frame found across tasks in Experiment 2 were not due to differences in stimulus familiarity (Rock, 1973, 1986).

\section{GENERAL DISCUSSION}

In three experiments, we examined the relative influence of task and stimulus properties on the spatial frame of reference in the perception of forms rotated in the frontal plane. Experiment 1 compared object-naming performance and mirror-normal discrimination of letters and digits. The frame for naming was aligned more closely with retinal directions, whereas the frame for mirror-normal discrimination was aligned more closely with environmental directions. Experiment 2 demonstrated that these results were not due to differences between the stimulus sets used in the two tasks. When the same objects were named and discriminated on the basis of left-right reflection, the results of Experiment 1 were upheld. The final experiment demonstrated that the results of Experiment 2 were not due to differences in familiarity with the stimuli used in the two tasks. Wide ranges of concept familiarity and complexity (Snodgrass \& Vanderwart, 1980) were also available with the objects presented for both tasks, suggesting that the results were not dependent on any particular level of these factors (see Corballis et al., 1976; Rock, 1973, 1974; Rock \& DiVita, 1987). Overall, the results of the naming and discrimination tasks clearly indicate that the orientation of the subjective frame of reference in the perception of rotated forms is determined primarily by the nature of the task rather than the nature of the stimuli.

The retinally aligned reference frame demonstrated with object naming contrasts with the environmentally aligned reference frame suggested in the recognition of disoriented nonsense or fragmented forms (Rock \& Heimer, 1957). The orientation-sensitive mechanisms involved in recognition memory, including the frame of reference, ought to be the same as those involved in naming, provided that both tasks access the same long-term representations. However, it is possible that some recognition-memory tasks only access an "episodic" trace.

Alternatively, the discrepancy in reference frames reported for naming and recognition memory could be due to a procedural feature of the memory studies. Without instructions explicitly stating that the forms in the recognition session of the memory experiment were rotated, the subjects probably assumed that they were environmentally upright, particularly since they were assumed to be oriented that way in the learning session (Rock \& Heimer, 1957). Also, since the stimuli were nonsense forms known only on the basis of one previous presentation, when the forms were rotated in the recognition-session presentation, it may not have been possible to discern an intrinsic top or bottom that would have contradicted the assumption that they were environmentally upright. It would also have been difficult to discern the top and bottom of a rotated stimulus in the identification of rotated fragmented objects (Rock \& Heimer, 1957). Recognizing and/or identifying nonsense or fragmented forms under the assumption that all stimuli are environmentally upright may have given a spurious advantage to forms that were environmentally upright over forms that were tilted but retinally upright. This would result in apparent support for an environmental reference frame.

In fact, the present results are in accord with one of Rock's early studies on the identification of fragmented objects (Rock \& Heimer, 1957, Experiment 6). When the subjects in that experiment were informed that the objects would be tilted in the same direction as their heads, identification was actually better when the stimuli were retinally upright than when they were environmentally upright (and retinally disoriented). These results are consistent with the use of a retinally aligned frame. Knowledge that an object is rotated is important for its recognition or identification (Rock, 1973), possibly so that the top of the object can be aligned with the top of the reference frame. However, specific orientation information is no more useful than general information that the upcoming stimulus is rotated (Braine, 1965).

It is unlikely that this difficulty in realizing that forms are tilted would occur with intact, familiar objects viewed for an unlimited amount of time, even when subjects are not informed that they will see tilted forms. No spurious advantage for environmentally upright stimuli was therefore likely in the present naming task, especially since the subjects knew that they would see rotated forms. In general, the results of the present and previous studies suggest that when the viewer is aware that the stimuli can be rotated, the frame of reference is retinal for both naming and recognition tasks (see Rock \& Heimer, 1957).

What aspects of the naming and discrimination tasks could underlie the use of different spatial reference frames? Corballis and Beale (1976) have suggested that 
we assign left and right on the basis of reference to our own body. The present results suggest that we further map bodily left and right onto environmental left and right, perhaps because unlike the body, the environment is a fixed and therefore more reliable reference. The results presented here indicate that both of the discrimination tasks require relating directions of an object or pattern to directions assigned to external space (left vs. right). It is not obvious why this would be a requirement of object naming. Without this requirement, the default reference frame may be retinal.

Despite the finding that different frames of reference are used in naming and left-right reflection discriminations, the same rotational alignment process may be used during performance of these tasks. The slopes of the response functions for stimuli disoriented between $0^{\circ}$ and $120^{\circ}$ are very similar for the two tasks (Jolicoeur, 1985 , 1988). This result suggests that the alignment of the top of the stimulus with the top of the reference frame is carried out at similar rates in the two tasks. Implicit here is the notion that the same rotational processes operate within a common reference frame that is positioned in different initial orientations depending on the task.

Two interpretations of the average subjective reference frame are possible. One interpretation sees the frame as perfectly retinally aligned or perfectly environmentally aligned on any one trial, with the frame on average across trials more frequently retinal or environmental. The other interpretation sees the frame as neither perfectly retinal nor perfectly environmental on any one trial, but rather somewhere between the two. The frame is on average, then, more closely aligned with retinal or environmental directions. Our results do not allow us to favor either interpretation. However, like Corballis, Anuza, et al. (1978), we believe that the latter interpretation has more intuitive appeal because "we sometimes operate within environments which are tilted with respect to both gravity and the axes of our heads, as in a tilted vehicle or in reading a tilted page"' (p. 283).

These experiments have demonstrated that the orientation of the frame of reference used during the performance of a task can elucidate the underlying processes of the task. The spatial referencing processes involved in naming a rotated object are different from those involved in determining the left-right reflection of the object. It will be of future interest to determine if the spatial demands of left-right reflection discriminations are somewhat unique, or if other tasks that are sensitive to stimulus orientation demonstrate the same reference to external space.

\section{REFERENCES}

AtTNeave, F., \& Olson, R. K. (1967). Discriminability of stimuli varying in physical and retinal orientation. Journal of Experimental Psychology, 74, 149-157.

Attreave, F, \& Reid, K. W. (1968). Voluntary control of frame of reference and shape equivalence under head rotation. Journal of $E x$ perimental Psychology, 78, 153-159.

Braine, L. G. (1965). Disorientation of forms: An examination of Rock's theory. Psychonomic Science, 3, 541-542.
COOPER, L. A., \& Shepard, R. N. (1973). Chronometric studies of the rotation of mental images. In W. G. Chase (Ed.), Visual information processing (pp. 75-176). New York: Academic Press.

Corballis, M. C. (1988). Recognition of disoriented shapes. Psychological Review, 95, 115-123

Corballis, M. C., Anuza, T., Blake, L. (1978). Tachistoscopic perception under head tilt. Perception \& Psychophysics, 24, 274. 284.

Corbalus, M. C., Beale, I. (1976). The psychology of lefi and right Hillsdale, NJ: Erlbaum.

Corballis, M. C., \& Nagourney, B. A. (1978). Latency to categorize disoriented alphanumeric characters as letters or digits. Cana dian Journal of Psychology, 32, 186-188.

Corbalus, M. C., Nagourney, B. A., Shetzer, L. I., a Stefanatos, G (1978). Mental rotation under head tilt: Factors influencing the location of the subjective reference frame. Perception \& Psychophysics, 24, 263-273

Corballis, M. C., \& Roldan, C. E. (1975). Detection of symmetry as a function of angular orientation. Journal of Experimental Psychology: Human Perception \& Performance, 1, 221-230.

Corballus, M. C., Zerodoff, J., \& Roldan, C. E. (1976). What's up in mental rotation? Perception \& Psychophysics, 19, 525-530.

Corballis, M. C., Zerodoff, N. J., Shetzer, L. I., Butler, P. B. (1978). Decisions about identity and orientation of rotated letters and digits. Memory \& Cognition, 6, 98-107.

EI.EY, M. G. (1982). Identifying rotated letter-like symbols. Memory \& Cognition, 10, 25-32.

Hinton, G. E., Parsons, L. M. (1981). Frames of reference and mental imagery. In A. Baddeley \& J. Long (Eds.), Attention and performance (Vol. 9, pp. 261-277). Hillsdale, NJ: Erlbaum.

Hock, H. S., \&ullivan, M. (1981). Alternative spatial reference systems: Intentional vs. incidental learning. Perception \& Psychophysics, 29, 467-474.

Jolicoeur, P. (1985). The time to name disoriented natural objects. Memory \& Cognition, 13, 289-303

Jolicoeur, P. (1988). Mental rotation and the identification of disoriented objects. Canadian Joumal of Psychology, 42, 461-478.

Jolicoeur, P., \& MILLIKEN, B. (1989). Identification of disoriented objects: Effects of context of prior presentation. Journal of Experimental Psychology: Learning, Memory, \& Cognition, 15, 200-210.

Jolicoeur, P., Snow, D., \& Murray, J. (1987). The time to identify disoriented letters: Effects of practice and font. Canadian Journal of Psychology, 41, 303-316

KORIAT, A., NORMAN, J. (1984). What is rotated in mental rotation? Journal of Experimental Psychology: Learning. Memory, \& Cognition, 10, 421-434.

MAKI, R. H. (1986). Naming and locating the tops of rotated pictures Canadian Journal of Psychology, 40, 368-387.

Miller, E. F. (1962). Counterrolling of the human eyes produced by head tilt with respect to gravity. Acta Otolaryngologica, 54, 479-501.

Palmer, S. (1985). The role of symmetry in shape perception. Acta Psychologica, 59, 67-90

Robertson, L. C., Palmer, S. E. \& Gomez, L. M. (1987). Reference frames in mental rotation. Joumal of Experimental Psychology. Learning, Memory, \& Cognition, 13, 368-379.

Rock, I. (1956). The orientation of forms on the retina and in the environment. American Joumal of Psychology, 69, 513-528.

Rock. I. (1973). Orientation and form. New York: Academic Press. Rock, I. (1974). The perception of disoriented figures. Scientific American, 230, 78-85.

Rock, I. (1986). The description and analysis of object and event perception. In K. R. Boff, L. Kaufman, \& J. P. Thomas (Eds.), Handbook of perception and human performance: Vol. 2. Cognitive processes and performance (p. 33-24). New York: Wiley.

Rock, I., \& DiVITA, J. (1987). A case of viewer-centered object perception. Cognitive Psychology, 19, 280-293.

Rock, I., \& HEimer, W. (1957). The effect of retinal and phenomenal orientation on the perception of form. American Journal of Psychol ogy, 70, 493-511.

SHWARTZ, S. P. (1981). The perception of disoriented complex objects. Unpublished manuscript, Yale University, New Haven, CT. 
Snodgrass, J. G., \& VANDerwart, M. (1980). A standardized set of 260 pictures: Norms for name agreement, image agreement, familiarity, and visual complexity. Journal of Experimental Psychology: $\mathrm{Hu}$ man Learning \& Memory, 6, 174-215.

WHITE, M. J. (1980). Naming and categorization of tilted alphanumeric characters do not require mental rotation. Bulletin of the Psychonomic Society, 15, 153-156.

\section{NOTES}

1. Some debate has focused on whether the image is rotated to the position of the frame (Cooper \& Shepard, 1973; Koriat \& Norman, 1984) or the frame is rotated to the position of the image (Robertson et al., 1987). Our interpretation of the current data does not address this issue or rely upon either view.

2. We use the term "environmentally upright" to denote a direction that is upright relative to all aspects of the environment, including gravity. We do not attempt to evaluate the relative contribution of different en- vironmental cues, be they visual, proprioceptive, or vestibular, to the subjective assignment of this direction.

3. Although hesitation errors such as "ah" and premature tripping of the voice key are not true errors, their inclusion with the error rates should not have adversely affected the pattern of error rates across conditions. Trials that were spoiled in these ways were not systematic and should have been randomly distributed across all conditions.

4. All of the reported analyses for the naming task are based on responses to the complete set of 120 items presented. Results from this complete set of items are reported because they should represent the most stable estimates of the parameters in each condition of this task. However, the left-right discrimination task used a subset of these items, so item set could be responsible for any differences found between the two tasks. For this reason, all of the analyses reported for the naming task were also performed on the 72-item subset used in the left-right discrimination task. All of the effects found that were significant with the complete set of items were also found with the 72-item set. Similarly, all of the effects that failed to reach significance with the 120 -item set were not significant with the 72 -item set. Differences between the tasks must therefore be due to the tasks and not to the item sets.

\section{APPENDIX A}

Line drawings of Items 1-120 were used as stimuli in the naming tasks in Experiments 1 and 2. Line drawings of Items 1-72 were used in the left-right discrimination task in Experiments 2 and 3. The drawings can be seen in Snodgrass and Vanderwart (1980). The drawing of the eagle was altered so that its head faced in the same direction as its body. For each item, the most frequently used label is listed first (Snodgrass \& Vanderwart, 1980). Labels in parentheses were considered acceptable alternatives in the current experiments.

$\begin{array}{ll}\text { 1. Airplane (Plane, Jet) } & \text { 31. Gorilla (Ape, Orangutan) } \\ \text { 2. Alligator (Crocodile, Lizard) } & \text { 32. Grasshopper } \\ \text { 3. Ant } & \text { 33. Gun (Pistol, Revolver) } \\ \text { 4. Baby Carriage (Pram, } & \text { 34. Helicopter (Chopper) } \\ \text { Stroller) } & \text { 35. Hen (Chicken) } \\ \text { 5. Barn (Farm) } & \text { 36. Horse } \\ \text { 6. Bear (Polar Bear) } & \text { 37. Iron } \\ \text { 7. Bee (Wasp, Bumblebee) } & \text { 38. Ironing Board (Ironing Table) } \\ \text { 8. Beetle (Cockroach, Roach) } & \text { 39. Kangaroo } \\ \text { 9. Bicycle (Bike) } & \text { 40. Kettle (Tea Kettle, Tea Pot) } \\ \text { 10. Bird (Sparrow) } & \text { 41. Leopard (Cheetah) } \\ \text { 11. Boot } & \text { 42. Lion } \\ \text { 12. Bus } & \text { 43. Monkey (Chimp, } \\ \text { 13. Camel (Dromedary) } & \text { Chimpanzee) } \\ \text { 14. Car (Automobile) } & \text { 44. Motorcycle (Bike) } \\ \text { 15. Cat } & \text { 45. Mouse (Rat) } \\ \text { 16. Church (Chapel) } & \text { 46. Ostrich (Emu) } \\ \text { 17. Cow } & \text { 47. Peacock } \\ \text { 18. Deer (Stag, Reindeer, } & \text { 48. Penguin } \\ \text { Antelope) } & \text { 49. Pig } \\ \text { 19. Dog } & 50 . \text { Rabbit (Bunny) } \\ \text { 20. Donkey (Mule) } & 51 \text {. Racoon } \\ \text { 21. Duck } & 52 . \text { Rhinoceros (Rhino) } \\ \text { 22. Eagle (Hawk, Falcon) } & 53 . \text { Roller Skate (Skate) } \\ \text { 23. Elephant } & 54 . \text { Rooster } \\ \text { 24. Fly } & 55 . \text { Sailboat (Ship, Boat) } \\ \text { 25. Foot } & 56 . \text { Seahorse } \\ \text { 26. Football Helmet (Helmet) } & 57 . \text { Seal (Sea Lion) } \\ \text { 27. Fox (Wolf) } & 58 . \text { Sheep (Lamb) } \\ \text { 28. Frog } & 59 . \text { Shoe } \\ \text { 29. Giraffe } & 60 . \text { Skunk } \\ \text { 30. Goat } & 61 . \text { Sled (Sleigh) } \\ & \end{array}$

31. Gorilla (Ape, Orangutan)

32. Grasshopper

35. Hen (Chicken)

36. Horse

37. Iron

39. Kangaroo

41. Leopard (Cheetah)

42. Lion

3. Monkey (Chimp, Chimpanzee)

45. Mouse (Rat)

46. Ostrich (Emu)

47. Peacock

48. Penguin

49. Pig

50. Rabbit (Bunny)

51. Racoon

52. Rhinoceros (Rhino)

53. Roller Skate (Skate)

54. Rooster

55. Sailboat (Ship, Boat)

56. Seahorse

57. Seal (Sea Lion)

59. Shoe

61. Sled (Sleigh)
62. Snail

63. Spider

64. Squirrel

65. Swan

66. Tiger

67. Train (Locomotive)

68. Truck (Lorry)

69. Turtle (Tortoise)

70. Wagon

71. Watering Can (Sprinkler, Water Can)

72. Zebra

73. Accordion

74. Apple

75. Ashtray

76. Basket (Picnic Basket)

77. Bed

78. Blouse (Shirt)

79. Bottle (Wine Bottle)

80. Bowl

81. Cake

82. Candle

83. Cannon

84. Caterpillar

85. Chair

86. Coat (Overcoat, Jacket)

87. Crown

88. Cup (Tea Cup)

89. Desk

90. Doll (Baby Doll)

91. Dress
92. Dresser (Chest of Drawers, Bureau, Drawers)

93. Frying Pan (Pan)

94. Garbage Can (Trash Can)

95. Glass (Cup)

96. Harp

97. Hat (Fedora)

98. House

99. Jacket (Shirt, Coat)

100. Lamp (Light)

101. Mushroom

102. Nose

103. Owl

104. Piano (Grand Piano)

105. Pitcher (Jug)

106. Pot (Saucepan)

107. Record Player (Stereo, Turntable)

108. Rocking Chair (Chair)

109. Shirt

110. Snowman

111. Sofa (Couch, Chesterfield)

112. Spinning Wheel

113. Stool

114. Stove (Oven)

115. Sweater (Pullover, Shirt)

116. Swing

117. Telephone (Phone)

118. Tree

119. Well (Wishing Well)

120. Wine Glass (Glass, Goblet) 


\section{APPENDIX B}

Predicted pattern of response times across orientation when a perfectly retinally aligned frame of reference is used.

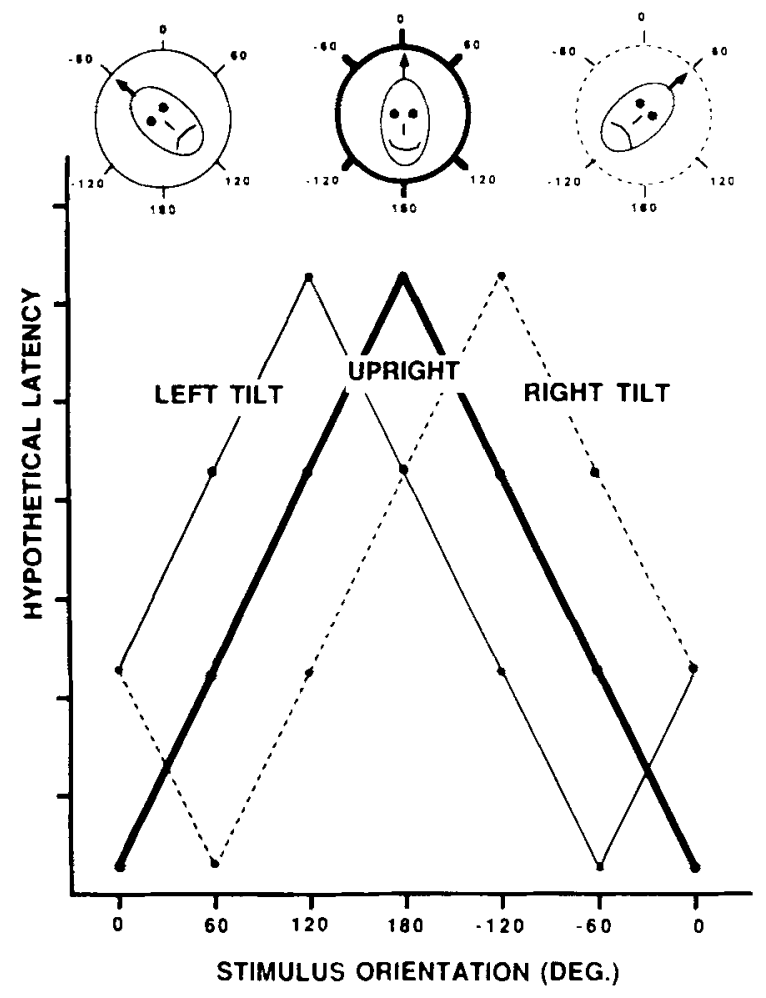

\section{APPENDIX C}

Predicted pattern of response times across orientation when a perfectly environmentally aligned frame of reference is used.

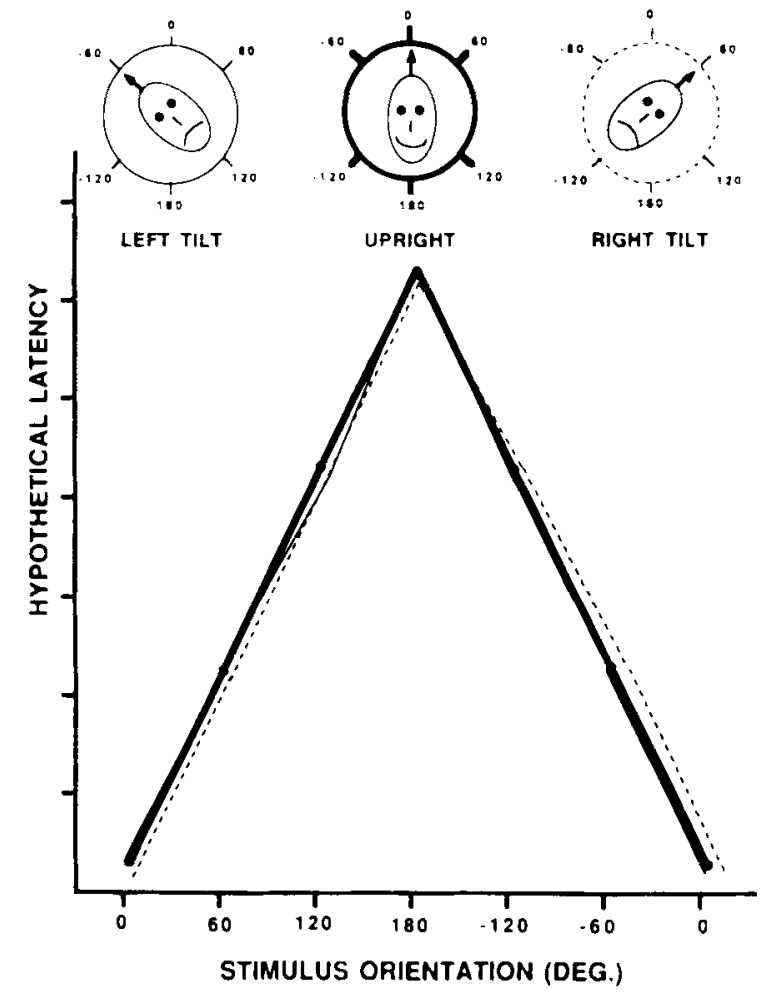

(Manuscript received March 30, 1988;

revision accepted for publication June 2, 1989.) 